\title{
Compromise in CDMA Network Planning
}

\author{
Stephen Hurley, Leigh Hodge \\ School of Computer Science, Cardiff University, Cardiff, UK \\ Email:steve@cs.cf.ac.uk \\ Received June 7, 2010; revised August 16, 2010; accepted August 20, 2010
}

\begin{abstract}
CDMA network planning, for example in 3G UMTS networks, is an important task whether for upgrading existing networks or planning new networks. It is a time consuming, computationally hard, task and generally requires the consideration of both downlink and uplink requirements. Simulation experiments presented here suggest that if time is a major consideration in the planning process then as a compromise only uplink needs to be considered.
\end{abstract}

Keywords: CDMA, Network Planning, Optimization, Simulation

\section{Introduction}

The past decade has seen the emergence of many computational approaches for cellular network site selection, configuration and dimensioning. Many of these contributions have paid attention to planning wide-area FTDMA systems such as second generation GSM where planning is generally carried out using a downlink transmission model and independent criteria for coverage and capacity, e.g [1].

A number of researchers have considered the rather more complex problem of network planning for UMTS networks. Amaldi et al ([2-5]) propose a mathematical programming model which accounts for both the uplink and downlink directions as well as for base station configuration issues including location, height, tilt and azimuth. To allow solutions to be sought in reasonable time, approximate solutions are sought via application of the tabu search meta-heuristic. In [6] and [7], Zhang, Yang, et al. propose a mathematical framework for UMTS network planning that considers fast power control, soft handover and pilot signal power in the uplink and downlink directions. Again, solutions are sought via the application of meta-heuristics (SA and evolutionary SA). Ben Jamaa et al. ([8,9]] propose an approach which employs a multi-objective GA (MOGA) to simultaneously optimize capacity and coverage by adjusting antenna parameters and common channel transmitted powers (antenna locations fixed). A multi-objective fitness function is employed which can consider objectives such as coverage, capacity and cost. The result of the MOGA is a Pareto set of non-dominated solutions.

For third generation systems such as UMTS, the planning problem is significantly more complex than for FTDMA systems due to the dependency between capacity and coverage. The underlying CDMA protocol requires that on each link, a target signal to noise ratio (SNIR) is maintained, and consequently per-link power allocation is required before user service coverage and cell load can be accurately assessed. However, determining this is non-trivial as one user transmission is seen as interference by all other users, making coverage/capacity evaluation sensitive to other users. Transmission power minimization is important and the real-time UMTS system achieves this by fast power control. However for modeling purposes, this is costly to repeatedly simulate because all links are required to frequently re-evaluate their SNIR and adjust power accordingly.

Whitaker R. M. et al describe two efficient heuristic algorithms that enable the evaluation of service coverage and cell loading in both the uplink and downlink directions. In this paper, we investigate the application of these heuristics to the problem of cell planning for UMTS networks. The cell planning problem (CPP) is concerned with the selection of antennae from a set of candidate antennae, and the configuration of these antennae, such that an optimal configuration is achieved. As for the frequency assignment problem (FAP), the CPP has $N P$-complete computational complexity. This dictates that exact solutions to the CPP cannot be attained in practice. Hence we consider a meta-heuristic optimization approach.

The remainder of this paper is organized as follows. Section 2 describes the model and Section 3 provides a brief overview of the uplink and downlink service coverage/load evaluation heuristics. Section 5 outlines the 
optimization problem and the meta-heuristic employed. A number of test problems are defined in Section 5 and the results and analysis of applying our optimization approaches to these problems can be seen in Section 6 .

\section{Model}

The uplink (UL) and downlink (DL) dedicated channels and the pilot signal is included in our model. Parameters are described in Table 1, Table 2 and Table 3 and are defined relative to the link direction under consideration.

The terms cell, antenna and transmitter are used interchangeably when describing aspects of coverage. A number of candidate antenna locations are defined for a given region. A planning/optimization process is employed to select and configure antennae based on defined objectives. Discrete test points from the region are used to sample service coverage. Each test point is a physical position (expressed in two dimensional Cartesian co-ordinates). Two types of test point are defined in our

Table 1. Global parameters.

\begin{tabular}{cl}
\hline Symbol & Description \\
\hline$R_{i}$ & CDMA chip rate. \\
$S_{A}$ & Data rate for service $i$. \\
$S_{p t p}$ & Set of all antennas in the working region. \\
$S_{S t p}$ & The set of covered pilot test points. \\
Ostp $_{\text {stp }}$ & The set of service test points. \\
\hline
\end{tabular}

Table 2. Uplink parameters.

\begin{tabular}{cl}
\hline Symbol & Description \\
\hline$p_{x y}^{U L}$ & Received power from stp $x$ at a cell $y$. \\
$I_{\text {own }}$ & Total received power from stp active in cell $y$. \\
$I_{\text {oth }}$ & Total received power from stp active in cells other than $y$. \\
$I_{y}$ & Total received power from all active stp. \\
$N^{*}$ & Noise power seen at the antennas receiver in an empty cell. \\
$\left(E_{b} / N_{o}\right)_{U L}$ & Target threshold for $E_{b} / N_{o}$ ratio at an stp for the dedicated UL channel (service dependent). \\
$\eta_{U L, y}$ & Uplink load at cell $y$. \\
\hline
\end{tabular}

Table 3. Downlink parameters.

\begin{tabular}{cl}
\hline Symbol & Description \\
\hline Iown & Total power received from serving cell (all links and pilot). \\
Ioth & Total power received from all cells other than the serving cell. \\
$\alpha$ & Orthogonality Factor. \\
$P$ & Noise power (thermal and equipment) seen at a test point. \\
$p_{x y}$ & Power allocated by cell $y$ for stp $x$ as received at stp $x$. \\
$p_{x y}$ ilot & Pilot power from cell $y$ as received at stp $x$. \\
$\left(E_{c} / I\right)_{p i l o t}$ & Target threshold for pilot $E_{c} / I$ ratio. \\
$\left(E_{b} / N_{o}\right)_{U L}^{*}$ & Target threshold for $E_{b} / N_{o}$ ratio at an stp for a dedicated DL channel (service dependent). \\
$\eta_{D L, y}$ & Downlink load at cell $y$. \\
PtxTotal $y$ & Total of allocated transmit powers in cell $y$. \\
Ptxmax $y$ & Maximum transmit capability of cell $y$. \\
$\eta_{\text {pilot, } y}$ & Proportion of Ptxmax $y$
\end{tabular}


model: service test points (stp) and pilot test points (ptp). The ptp are used to assess pilot signal quality. At an stp, quality of both UL and DL dedicated channels are as sessed for a particular service, which is defined prior to evaluation.

\subsection{Test Point Coverage and Cell Load}

The pilot signal is transmitted at a proportion $\eta_{\text {pilot, } y}$ of the maximum cell power. A ptp $x$ is served by antenna $y$ when the received energy per chip relative to the total spectral density Ec/Io at least meets the target $E c / I o_{\text {pilot }}$. Letting $I_{y}=I_{\text {own }}+I_{\text {oth }}$, then $x$ is served if and only if:

$$
\frac{p_{x y}^{\text {pilot }}}{N+I_{y}} \geq\left(E_{c} / I_{o}\right)_{\text {pilot }}
$$

An stp is covered in a particular link direction if energy per bit relative to spectral noise density (Eb/No) at least meets the required target threshold. For an stp $x$ connected to antenna $y, x$ is UL covered if and only if:

$$
\frac{W}{R_{i}} \cdot \frac{p_{x y}^{U L}}{I_{y}-p_{x y}+N} \geq(E b / N o)_{U L}^{*}
$$

In the downlink, for an stp $x$ and serving antenna $y, x$ is $D L$ covered if and only if:

$$
\frac{W}{R_{i}} \cdot \frac{p_{x y}^{D L}}{I_{\text {own }}(1-\alpha)+I_{\text {oth }}+p_{n}} \geq(E b / N o)_{D L}^{*}
$$

There are various ways in which cell loading can be assessed. Wideband power-based measurement is used in this model because it directly identifies the resources being allocated. The downlink load at cell $y$ is estimated by:

$$
\eta_{D L, y}=\frac{\text { PtxTotal }_{y}}{\text { Ptxmax }_{y}}
$$

while the uplink load at cell $y$ is estimated by:

$$
\eta_{U L, y}=\frac{I_{y}}{I_{y}+N}
$$

Note that a ptp's ability to be served depends on downlink cell load. Consequently a $p t p$ is covered if and only if it is served when all cells $y$ are operating at maximum permitted downlink load $\eta_{\mathrm{DL}, \mathrm{y}}^{\max }$. Covered $p t p$ can see the pilot signal independent of traffic and are collectively denoted $S_{p t p \text {. }}$

To ensure that an stp can see the pilot signal, it is required that $S_{s t p} \subseteq S_{p t p}$. A list $O_{s t p}$ of the set $S_{s t p}$ is also required to specify the order in which stp are prioritized for admission. The ordering is defined based on the received signal strength from the best serving antenna with those with the strongest signal given priority.

\section{Evaluation Heuristics}

Calculating off-line transmission power for target $\mathrm{Eb} / \mathrm{No}$ attainment on a link requires knowledge of interference levels or equivalently cell loads. However, interference/cell loads depend on per-link transmission powers. This dependency has led to the analytical characterization of the problem [11]. We employ an algorithmic approach which initially over-estimates interference/cell loading and then uses a feedback mechanism to iteratively update and reduce the conservative error. When this feedback mechanism is applied, the heuristic can converge to a state where inaccuracy in power allocation and cell loading is negligible. From this, stp coverage and cell loads can be directly obtained.

Detailed discussion of the uplink and downlink evaluation heuristics used here can be found in [10].

\section{Optimization Problem}

It is assumed that for optimization, the objective is to select/activate and configure (where appropriate) a subset of antennae from the set of candidate antennae such that coverage is maximized for a specified number of active transmitters. After experimentation with a number of meta-heuristics it was determined that tabu search (TS) was the most effective approach for this optimization problem. The TS algorithm employed is summarized in Figure 1. A detailed description of the TS meta-heuristic can be found in [12].

The operation of our tabu search approach can be characterized by the following components: starting configuration; moves; evaluation type and cost function.

\subsection{Starting Configuration}

The starting configuration can impact on the final configuration achieved by the TS. Having investigated a number of starting configurations (i.e., all transmitters inactive, all transmitters active, random transmitters active and Halton configuration - approximately random uniformly distributed) it has been shown that whilst the

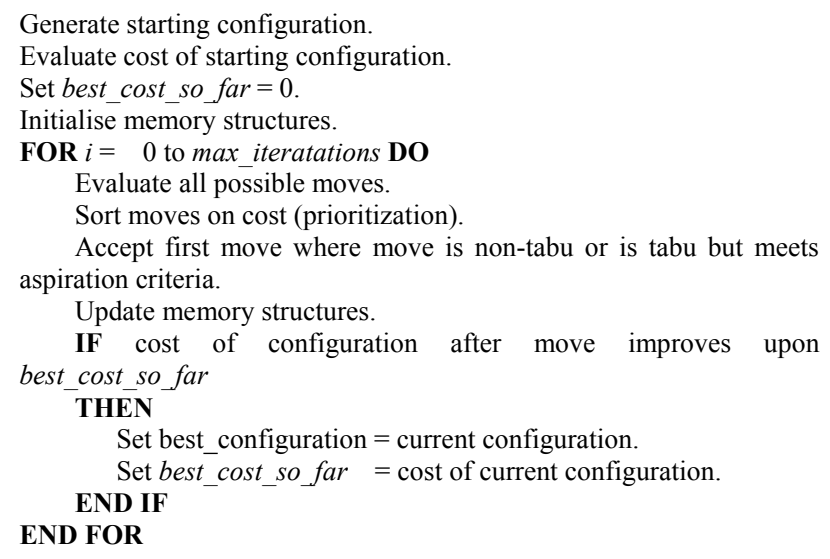

Figure 1. Generic tabu search algorithm 
effectiveness of each starting configuration is dependent on the problem scenario and other parameter settings, starting with all transmitters inactive leads to the best solutions in general.

\subsection{Moves}

A range of different moves are employed by the TS. At each iteration of the TS, the impact of each of the available moves is evaluated by applying the move to each candidate antenna in order to determine the best possible move at that instance. The quality of each move is determined by the cost function. A range of moves have been implemented from which a subset of moves can be selected for evaluation:

- Activate an inactive transmitter i.e. make operational.

- Deactivate an active transmitter i.e. shut down.

- Swap transmitters:

- Deactivate an active transmitter and activate a randomly selected inactive transmitter .

- Activate an inactive transmitter and deactivate a randomly selected active transmitter .

- Determine the best azimuth for a transmitter - the azimuth for a given transmitter is varied (controlled by azimuth_increment) such that all available azimuth configurations in the sector are evaluated. The configuration with the best cost can then be determined.

- Determine the best tilt for a transmitter - the tilt for a given transmitter is varied (controlled by tilt_increment) such that all available tilt configurations in the range tilt_max to tilt_min are are evaluated. The configuration with the best cost can then be determined.

\subsection{Evaluation Type}

When evaluating moves, cost functions are employed in conjunction with an evaluation type. The evaluation type defines the evaluation heuristic used to determine service (i.e. uplink or downlink evaluation heuristic) and any constraints on the feedback mechanism employed to determine total load. In its least constrained form the iterative feedback mechanism repeats until the variation in the load is less than a predefined threshold. Iterating to convergence may require much iteration. This is time consuming and additional iterations achieve decreasing returns. As a result, in order to achieve an acceptable runtime for the TS (which need to perform large numbers of evaluations) the number of feedback iterations is constrained when employed for evaluating moves, only running to convergence for the final TS solution.

\subsection{Cost Function}

TS requires that a cost is associated with problem configurations such that an optimal or near optimal configuration can be sought. A weighted cost function is employed to enable the following objectives to be considered:

1) Meet the constraint on the number of transmitters.

2) Maximise coverage.

3) Favour configurations with lower total loads.

The tabu search seeks to minimize the total cost of a configuration, defined as:

$$
\begin{aligned}
& \text { total_cost }=\left(\operatorname{covg} \_c o s t * w_{c}\right)+\left(\operatorname{actv} \_c o s t * w_{a}\right)+ \\
& \left(l d \_c o s t * w_{l}\right)
\end{aligned}
$$

where $w_{c}, w_{a}$ and $w_{l}$ are weightings for coverage cost, active cost and load cost respectively.

Coverage cost is defined as

$$
\text { covg_cost }=100-\text { coverage }
$$

where coverage is the percentage of stp that are covered in the downlink or uplink direction.

$$
\text { Active cost is defined as }
$$

$$
\text { actv_cost }=\text { trans_thrs }- \text { active_trans }
$$

where trans_thrs is the desired number of active transmitters and active trans is the number of active transmitters.

Load cost is defined as

$$
\text { ld_cost }=\sum \eta_{D L}
$$

for all active transmitters in the downlink direction and

$$
\text { ld_cost }=\sum \eta_{U L}
$$

for all active transmitters in the uplink direction.

It should be noted that as some objectives are competing (e.g. coverage and total load) it may not be possible to determine the configuration which exhibits the optimal trade-off between objectives. This would require a more complex (and time consuming) multi-objective approach.

\section{Experimentation}

The purpose of experimentation is to compare the performance of network configurations generated using uplink and downlink evaluation heuristics. Performing optimization for both link directions is time consuming. Consequently, it would be useful if we could identify an optimization configuration that provides a good trade-off between optimizing for uplink and for downlink, i.e., a single approach that produces network configurations that perform well in both link directions. Whilst this trade-off may not be acceptable when producing final configurations, it could be beneficial in preliminary stages of network planning where some accuracy can be traded for the decreased evaluation time associated with optimizing for a single link direction.

\subsection{Test Problems}

All experiments consider a $3 \mathrm{~km}$ x $3 \mathrm{~km}$ transmission region containing 36 directional candidate antennae lo- 
cated at 12 uniformly distributed sites. Eight different problem scenarios have been considered as summarized in Table 4. Each scenario consists of a number of uniformly distributed stp with defined service requirements. Different scenarios have been generated by varying the number of stp considered and the distribution of services over these stp. Scenarios 5a, 5b and 5c have the same number of $s t p$ and number of $s t p$ with each service requirement, but a different distribution of these services over the stp. Signal attenuation is defined by the Hata path loss model.

\subsection{TS Configuration}

The tabu search was constrained to run for a maximum of 400 iterations and terminate after 50 iterations in which there is no improving move.

For each scenario and evaluation heuristic, two sets of moves are employed:

1) Activate/deactivate transmitter and swap transmitter activity (AS).

2) Activate/deactivate transmitter, swap transmitter activity, best tilt and best azimuth (ASBTBA) ${ }^{1}$.

These sets of moves were selected in order to investigate the impact of tuning the configuration of the antennae.

\section{Results}

In this section we present the results of optimization for each problem scenario. For each problem scenario, a number of problem instances are considered, each with different constraints on the maximum number of antennae allowed. Four optimization approaches are considered:

1) DL optimisation heuristics with AS

2) DL optimisation heuristics with ASBTBA

3) UL optimisation heuristics with AS

4) UL optimisation heuristic with ASBTBA

On completion of the TS the resulting configuration is evaluated for the opposite link direction, i.e.:
-DL optimize and evaluate configuration for DL and UL percentage coverage/service, and load.

-UL optimize and evaluate configuration for DL and UL percentage coverage/service, and load.

This enables us to determine how well configurations optimized in one link direction perform with respect to the opposite link direction. Further analysis is undertaken to determine:

1) Coverage Difference ('Covg Diff' column in the tables) - the difference between uplink and downlink coverage for each instance of a problem scenario. This gives an indication of how optimizing for one link direction impacts on the other.

2) Maximum DL Coverage Difference ('Max DL Covg Diff' column) - for each instance of a problem scenario the maximum percentage DL coverage ('Max \% DL Covg' column) for all optimization approaches is identified i.e. the maximum percentage DL coverage obtained from the DL or UL optimized AS or ASBTBA method. From this, the Maximum DL Coverage Difference is determined (by subtracting the coverage obtained for a problem instance from the maximum percentage DL coverage value), i.e. this indicates how well an optimization approach performs with respect to the best result. Consequently, this value gives an indication of which optimization approach performs best for each problem instance, and over all problem instances (based on the mean value).

3) Maximum UL Coverage Difference ('Max UL Covg Diff' column) - as Maximum DL Coverage Difference, but in the uplink direction.

\subsection{Sample Results}

Due to the volume of results generated from experimentation, only a subset of results is presented here ${ }^{2}$. For Problem 1, the results of all optimization approaches i.e. AS and ASBTBA are included (see Tables A1 to A4 in Appendix A). For other problems, the results for optimization approach ASBTBA are presented only (see Tables

Table 4. Problem scenarios.

\begin{tabular}{|c|c|c|c|c|c|c|c|}
\hline \multirow[b]{2}{*}{ Scenario } & \multicolumn{6}{|c|}{ No. stp assigned per service type } & \multirow[b]{2}{*}{ Total Capacity Req (kbps) } \\
\hline & No. stp & Pilot & $12.2 \mathrm{kbps}$ & $64 \mathrm{kbps}$ & $144 \mathrm{kbps}$ & $384 \mathrm{kbps}$ & \\
\hline 1 & 441 & 100 & 220 & 44 & 44 & 23 & 20,668 \\
\hline 2 & 441 & 147 & 0 & 294 & 0 & 0 & 18,816 \\
\hline 3 & 441 & 392 & 0 & 0 & 49 & 0 & 18,816 \\
\hline 4 & 961 & 630 & 220 & 44 & 44 & 23 & 20,668 \\
\hline $5 a, 5 b, 5 c$ & 961 & 299 & 440 & 88 & 88 & 46 & 41,336 \\
\hline 6 & 3721 & 1116 & 1675 & 372 & 372 & 186 & 169,235 \\
\hline
\end{tabular}

${ }^{1}$ For best tilt and best azimuth moves, an increment of 1 degree is applied.
${ }^{2} \mathrm{~A}$ complete set of results can be found in Appendix $\mathrm{B}$ at: www.cs.cf.ac.uk/bounds/documentation.htm 
Table 5. Analysis summary.

\begin{tabular}{|c|c|c|c|c|c|c|}
\hline Link Direction/ & Highest Mean & Highest Mean & Lowest & Lowest Mean & Lowest Mean & Lowest Combined \\
\hline Optimisation & DL Covg & UL Covg & Mean Covg & Max DL Covg & Max UL Covg & Mean Covg \\
\hline Method & & & Difference & Differnece & Difference & Difference \\
\hline DL AS & 1 & & 3 & 1 & & \\
\hline DL ASBTBA & $\begin{array}{l}2,3,4,5 a \\
5 b, 5 c, 6\end{array}$ & & & $\begin{array}{l}2,3,4,5 a \\
5 b, 5 c, 6\end{array}$ & & \\
\hline UL AS & & 3 & & 3 & & 2 \\
\hline UL ASBTBA & & $\begin{array}{l}1,2,3,4,5 a \\
5 b, 5 c, 6\end{array}$ & $\begin{array}{l}1,2,4,5 a \\
5 b, 5 c, 6\end{array}$ & & $\begin{array}{l}1,2,3,4,5 a \\
5 b, 5 c, 6\end{array}$ & $\begin{array}{l}1,3,4,5 a \\
5 b, 5 c, 6\end{array}$ \\
\hline
\end{tabular}

A5 to A18 in Appendix A) as these performs best in general (see Section 6.2).

\subsection{Analysis of Results and Conclusions}

In order to compare the effectiveness of the different optimization approaches, we examine for each scenario which approach gives the best performance with respect to a number of metrics:

1) highest mean DL \% coverage ('Highest Mean DL Covg' column);

2) highest mean UL \% coverage ('Highest Mean UL Covg' column);

3) lowest mean coverage difference ('Lowest Mean Covg Difference' column), i.e. for each scenario which method gives the lowest Coverage Difference value;

4) lowest mean maximum DL coverage difference (Lowest Mean Max DL Covg Difference' column) i.e. for each scenario which method gives the lowest mean Maximum DL Coverage Difference value;

5) lowest mean maximum UL coverage difference (Lowest Mean Max UL Covg Difference' column), i.e. as above but defined for UL, and

6) lowest combined (i.e., DL and UL) mean coverage difference ('Lowest Combined Mean Covg Difference' column) i.e. for each scenario which method gives the lowest value when adding the maximum DL and UL coverage difference means.

The results of this summary analysis can be seen in Table 5 which indicates the optimization method/ scenario combination that gives the best result for each of the above six metrics. As expected, the results show that including antenna configuration moves during optimization leads to increased coverage ${ }^{3}$. The results show that the DL ASBTBA optimization approach generally leads to the best downlink coverage and therefore the best i.e. lowest mean maximum DL coverage difference. Similarly the UL ASBTBA approach is consistently the best in terms of uplink.

Furthermore UL ASBTBA leads to the best (lowest) combined mean coverage difference i.e. for scenarios $1,3,4,5 \mathrm{a}, 5 \mathrm{~b}, 5 \mathrm{c}$ and 6 . This indicates that although optimization using the UL ASBTBA approach does not lead

${ }^{3}$ This is also confirmed by Amaldi et al in [2] who have also shown that it is preferable to simultaneously optimize antenna location and configuration than to do so separately. to the best levels of downlink coverage (though it is competitive in many places as illustrated by low mean maximum DL coverage difference values) it does result in the best overall combined mean coverage difference indicating that it performs better in terms of DL coverage than DL optimization does in terms of UL coverage. As a result, where time for planning a network is limited, the experimental results presented here suggest that a compromise in many cases is to optimize for uplink only rather than optimizing in both the uplink and downlink directions.

\section{Acknowledgements}

This work was funded by the UK's Engineering and Physical Science Research Council.

\section{References}

[1] S. Hurley, "Planning Effective Cellular Mobile Radio Networks," IEEE Transactions on Vehicular Technology, Cardiff, Vol. 51, No. 2, March 2002, pp 243-253.

[2] E. Amaldi, A. Capone, F. Malucelli and F. Signori, "Optimization Models and Algorithms for Downlink UMTS Radio Planning," Proceedings of Wireless Communications and Networking, Milan, Vol. 2, March 2003, pp. 827-831.

[3] E. Amaldi, A. Capone, F. Malucelli and F. Signori, "A Mathematical Programming Approach for W-CDMA Radio Planning with Uplink and Downlink Constraints," Proceedings of the Vehicular Technology Conference, 2003. Vol. 2, October 2003, pp. 806-810.

[4] E. Amaldi, A. Capone, F. Malucelli and F. Signori, "Radio Planning and Optimization of W-CDMA Systems," Lecture Notes in Computer Science - Personal Wireless Communications, Vol. 2775, 2003, pp. 437-447.

[5] E. Amaldi, A. Capone and F. Malucelli, "Radio Planning and Coverage Optimization of 3G Cellular Networks," Wireless Networks, Vol. 14, No. 4, August 2008, pp. 435-447.

[6] J. Zhang, J. Yang, M. E. Aydin, and J. Y. Wu, "Mathematical Modelling and Comparisons of Four Heuristic Optimization Algorithms for WCDMA Radio Network Planning," Proceedings of the International Conference on Transparent Optical Networks, 2006, Vol. 3, June 2006, pp. 253-257. 
[7] J. Yang, M. E. Aydin, J. Zhang and C. Maple, "UMTS Base Station Location Planning: a Mathematical Model and Heuristic Optimisation Algorithms,"Communications, Vol. 1, No. 5, October 2007, pp. 1007-1014.

[8] S. B. Jamaa, Z. Altman, J. M. Picard and B. Fourestie, "Multi-objective Strategies for Automatic Cell Planning of UMTS Networks," Proceedings of the Vehicular Technology Conference, Vol. 4, May 2004, pp. 2420-2424.

[9] S. B. Jamaa, Z. Altman, J. M. Picard and B. Fourestie, "Combined Coverage and Capacity Optimisation for UMTS Networks," Telecommunications Network Strategy and Planning Symposium, Moulineaux June 2004, pp.
175-178.

[10] R. M. Whitaker, S. Allen and S. Hurley, "Efficient Offline Coverage and Load Evaluation for CDMA Network Modeling," IEEE Transactions on Vehicular Technology, Vol. 58, No. 7, August 2009, pp 3704-3712.

[11] L. Mendo and J. M. Hernando, "On Dimension Reduction for Next Generation Microcellular Networks," IEEE Transactions of Communications, Vol. 49, No. 2, 2001, pp. 243-248

[12] F. W. Glover and M. Laguna, "Tabu Search," Springer, 1997. 


\section{Appendix A}

Table A1. Scenario 1 - DL Optimised (AS).

\begin{tabular}{|c|c|c|c|c|c|c|c|c|c|c|}
\hline Num & Num & DL \% & $\mathrm{DL}$ & UL \% & $\mathrm{UL}$ & Covg & Max \% & Max DL & Max \% & Max UL \\
\hline $\mathrm{Tx}$ & Sites & Covg & Load & Covg & Load & Diff & DL Covg & Covg Diff & UL Covg & Covg Diff \\
\hline 15 & 12 & 97.7324 & 4.91592 & 93.424 & 6.92558 & 4.3084 & 97.9529 & 0.2205 & 97.5057 & 4.0817 \\
\hline 13 & 12 & 96.5986 & 3.85497 & 92.9705 & 6.77027 & 3.6281 & 97.5057 & 0.9071 & 96.6916 & 3.7211 \\
\hline 11 & 11 & 95.9184 & 4.51131 & 88.8889 & 5.65139 & 7.0295 & 96.6508 & 0.7324 & 93.424 & 4.5351 \\
\hline 9 & 9 & 93.424 & 3.67729 & 80.7256 & 4.75283 & 12.6984 & 93.424 & 0 & 89.5692 & 8.8436 \\
\hline 7 & 7 & 90.0227 & 3.61884 & 73.2426 & 4.2 & 16.7801 & 90.0227 & 0 & 85.6208 & 12.3782 \\
\hline Mean & & 94.73922 & & 85.85032 & & 8.8889 & & 0.372 & & 6.71194 \\
\hline
\end{tabular}

Table A2. Scenario 1 - DL Optimised (ASBTBA).

\begin{tabular}{|c|c|c|c|c|c|c|c|c|c|c|}
\hline Num & Num & DL \% & DL & UL \% & UL & Covg & $\operatorname{Max} \%$ & Max DL & $\operatorname{Max} \%$ & Max UL \\
\hline $\mathrm{Tx}$ & Sites & Covg & Load & Covg & Load & Diff & DL Covg & Covg Diff & UL Covg & Covg Diff \\
\hline 15 & 12 & 97.9529 & 5.45044 & 93.8776 & 6.90332 & 4.0753 & 97.9529 & 0 & 97.5057 & 3.6281 \\
\hline 13 & 12 & 97.5057 & 5.54612 & 90.7029 & 5.94145 & 6.8028 & 97.5057 & 0 & 96.6916 & 5.9887 \\
\hline 11 & 11 & 95.2381 & 4.56179 & 89.5692 & 5.69875 & 5.6689 & 96.6508 & 1.4127 & 93.424 & 3.8548 \\
\hline 9 & 9 & 92.7438 & 4.30398 & 85.7143 & 4.98792 & 7.0295 & 93.424 & 0.6802 & 89.5692 & 3.8549 \\
\hline 7 & 7 & 90.0227 & 3.69683 & 79.8186 & 3.9371 & 10.2041 & 90.0227 & 0 & 85.6208 & 5.8022 \\
\hline Mean & & 94.69264 & & 87.93652 & & 6.75612 & & 0.41858 & & 4.62574 \\
\hline
\end{tabular}

Table A3. Scenario 1 - UL Optimised (AS).

\begin{tabular}{|c|c|c|c|c|c|c|c|c|c|c|}
\hline Num & Num & DL $\%$ & $\mathrm{DL}$ & UL \% & $\mathrm{UL}$ & Covg & $\operatorname{Max} \%$ & Max DL & Max \% & Max UL \\
\hline$\overline{T x}$ & Sites & Covg & Load & Covg & Load & Diff & DL Covg & Covg Diff & UL Covg & Covg Diff \\
\hline 15 & 11 & 97.2789 & 4.23953 & 97.5057 & 8.27713 & 0.2268 & 97.9529 & 0.674 & 97.5057 & 0 \\
\hline 13 & 10 & 95.9184 & 3.75661 & 95.6916 & 7.2764 & 0.2268 & 97.5057 & 1.5873 & 96.6916 & 1 \\
\hline 11 & 11 & 94.7846 & 3.8032 & 93.424 & 6.22613 & 1.3606 & 96.6508 & 1.8662 & 93.424 & 0 \\
\hline 9 & 9 & 91.61 & 3.14089 & 89.5692 & 5.12837 & 2.0408 & 93.424 & 1.814 & 89.5692 & 0 \\
\hline 7 & 7 & 87.9819 & 2.88475 & 81.1791 & 4.09059 & 6.8028 & 90.0227 & 2.0408 & 85.6208 & 4.4417 \\
\hline Mean & & 93.51476 & & 91.47392 & & 2.13156 & & 1.59646 & & 1.08834 \\
\hline
\end{tabular}

Table A4. Scenario 1 - UL Optimised (ASBTBA).

\begin{tabular}{|c|c|c|c|c|c|c|c|c|c|c|}
\hline Num & Num & DL \% & DL & UL \% & UL & Covg & $\operatorname{Max} \%$ & Max DL & $\operatorname{Max} \%$ & Max UL \\
\hline $\mathrm{Tx}$ & Sites & Covg & Load & Covg & Load & Diff & DL Covg & Covg Diff & ULCovg & Covg Diff \\
\hline 15 & 12 & 97.5057 & 4.39257 & 97.5075 & 8.29146 & 0.0018 & 97.9529 & 0.4472 & 97.5075 & 0 \\
\hline 13 & 11 & 96.3719 & 4.33431 & 96.6916 & 7.24909 & 0.3197 & 97.5057 & 1.1338 & 96.6916 & 0 \\
\hline 11 & 9 & 96.6508 & 3.28007 & 93.1973 & 6.17624 & 3.4535 & 96.6508 & 0 & 93.424 & 0.2267 \\
\hline 9 & 9 & 91.1565 & 2.98996 & 89.1156 & 5.4 & 2.0409 & 93.424 & 2.2675 & 89.5692 & 0.4536 \\
\hline 7 & 7 & 89.1156 & 3.37209 & 85.2608 & 4.08703 & 3.8548 & 90.0227 & 0.9071 & 85.6208 & 0.36 \\
\hline Mean & & 94.1601 & & 92.35456 & & 1.93414 & & 0.95112 & & 0.20806 \\
\hline
\end{tabular}

Table A5. Scenario 2 - DL Optimised (ASBTBA).

\begin{tabular}{|c|c|c|c|c|c|c|c|c|c|c|}
\hline Num & Num & DL $\%$ & DL & UL \% & UL & Covg & $\operatorname{Max} \%$ & Max DL & $\operatorname{Max} \%$ & Max UL \\
\hline $\mathrm{Tx}$ & Sites & Covg & Load & Covg & Load & Diff & DL Covg & Covg Diff & UL Covg & Covg Diff \\
\hline 15 & 12 & 92.0635 & 7.76091 & 91.3832 & 8.12563 & 0.6803 & 92.0635 & 0 & 93.424 & 2.0408 \\
\hline 13 & 12 & 89.7959 & 6.94523 & 86.6213 & 7.26294 & 3.1746 & 89.7959 & 0 & 89.3424 & 2.7211 \\
\hline 11 & 11 & 85.7143 & 6.02062 & 82.7664 & 6.6 & 2.9479 & 85.7143 & 0 & 83.22 & 0.4536 \\
\hline 9 & 9 & 79.3651 & 4.92076 & 75.737 & 5.10716 & 3.6281 & 79.3651 & 0 & 77.3243 & 1.5873 \\
\hline 7 & 7 & 71.4286 & 3.97083 & 68.4807 & 4.1063 & 2.9479 & 71.4286 & 0 & 68.9342 & 0.4535 \\
\hline Mean & & 83.67348 & & 80.99772 & & 2.67576 & & 0 & & 1.45126 \\
\hline
\end{tabular}

Table A6. Scenario 2 - UL Optimised (ASBTBA).

\begin{tabular}{|c|c|c|c|c|c|c|c|c|c|c|}
\hline Num & Num & DL \% & DL & UL \% & UL & Covg & $\operatorname{Max} \%$ & Max DL & $\operatorname{Max} \%$ & Max UL \\
\hline $\mathrm{Tx}$ & Sites & Covg & Load & Covg & Load & Diff & DL Covg & Covg Diff & UL Covg & Covg Diff \\
\hline 15 & 12 & 90.4762 & 6.80249 & 93.424 & 8.68051 & 2.9478 & 92.0635 & 1.5873 & 93.424 & 0 \\
\hline 13 & 12 & 87.9819 & 5.60654 & 89.3424 & 7.8 & 1.3605 & 89.7959 & 1.814 & 89.3424 & 0 \\
\hline 11 & 11 & 83.22 & 5.42435 & 83.22 & 6.48371 & 0 & 85.7143 & 2.4943 & 83.22 & 0 \\
\hline 9 & 9 & 78.2313 & 4.23473 & 77.3243 & 5.3544 & 0.907 & 79.3651 & 1.1338 & 77.3243 & 0 \\
\hline 7 & 7 & 70.9751 & 3.65601 & 68.9342 & 4.16268 & 2.0409 & 71.4286 & 0.4535 & 68.9342 & 0 \\
\hline Mean & & 82.1769 & & 82.44898 & & 1.45124 & & 1.49658 & & 0 \\
\hline
\end{tabular}


Table A7. Scenario 3 - DL Optimised (ASBTBA).

\begin{tabular}{|c|c|c|c|c|c|c|c|c|c|c|}
\hline Num & Num & DL \% & DL & UL \% & UL & Covg & $\operatorname{Max} \%$ & Max DL & $\operatorname{Max} \%$ & Max UL \\
\hline $\mathrm{Tx}$ & Sites & Covg & Load & Covg & Load & Diff & DL Covg & Covg Diff & UL Covg & Covg Diff \\
\hline 15 & 12 & 98.8662 & 2.15203 & 99.5465 & 7.59356 & 0.6803 & 99.5465 & 0.6803 & 100 & 0.4535 \\
\hline 13 & 12 & 99.093 & 2.55291 & 99.093 & 5.97961 & 0 & 99.093 & 0 & 99.7732 & 0.6802 \\
\hline 11 & 10 & 98.6395 & 2.67339 & 98.1859 & 5.05613 & 0.4536 & 98.6395 & 0 & 99.093 & 0.9071 \\
\hline 9 & 9 & 97.9592 & 3.08148 & 97.2789 & 4.58512 & 0.6803 & 97.9592 & 0 & 97.9592 & 0.6803 \\
\hline 7 & 6 & 96.3719 & 2.44903 & 95.9184 & 3.82552 & 0.4535 & 96.5986 & 0.2267 & 96.5986 & 0.6802 \\
\hline Mean & & 98.18596 & & 98.00454 & & 0.45354 & & 0.1814 & & 0.68026 \\
\hline
\end{tabular}

Table A8. Scenario 3 - UL Optimised (ASBTBA).

\begin{tabular}{|c|c|c|c|c|c|c|c|c|c|c|}
\hline Num & Num & DL $\%$ & DL & UL \% & UL & Covg & Max \% & Max DL & Max \% & Max UL \\
\hline $\mathrm{Tx}$ & Sites & Covg & Load & Covg & Load & Diff & DL Covg & Covg Diff & UL Covg & Covg Diff \\
\hline 15 & 12 & 99.3197 & 2.45275 & 100 & 6.94763 & 0.6803 & 99.5465 & 0.2268 & 100 & 0 \\
\hline 13 & 11 & 99.093 & 2.50739 & 99.7732 & 6.52997 & 0.6802 & 99.093 & 0 & 99.7732 & 0 \\
\hline 11 & 10 & 98.1859 & 2.21099 & 99.093 & 5.88246 & 0.9071 & 98.6395 & 0.4536 & 99.093 & 0 \\
\hline 9 & 9 & 97.5057 & 2.25701 & 97.9592 & 5.01653 & 0.4535 & 97.9592 & 0.4535 & 97.9592 & 0 \\
\hline 7 & 7 & 96.5986 & 2.44812 & 96.5986 & 4.02627 & 0 & 96.5986 & 0 & 96.5986 & 0 \\
\hline Mean & & 98.14058 & & 98.6848 & & 0.54422 & & 0.22678 & & 0 \\
\hline
\end{tabular}

Table A9. Scenario 4 - DL Optimised (ASBTBA).

\begin{tabular}{|c|c|c|c|c|c|c|c|c|c|c|}
\hline Num & Num & DL \% & $\mathrm{DL}$ & UL \% & UL & Covg & $\operatorname{Max} \%$ & Max DL & Max \% & Max UL \\
\hline$\overline{T x}$ & Sites & Covg & Load & Covg & Load & Diff & DL Covg & Covg Diff & UL Covg & Covg Diff \\
\hline 15 & 12 & 98.231 & 3.48392 & 95.0052 & 5.71615 & 3.2258 & 98.3351 & 0.1041 & 98.231 & 3.2258 \\
\hline 13 & 12 & 98.4391 & 4.22513 & 96.0458 & 5.90235 & 2.3933 & 98.4391 & 0 & 97.9188 & 1.873 \\
\hline 11 & 10 & 97.8148 & 4.23715 & 92.82 & 4.91646 & 4.9948 & 97.8148 & 0 & 96.7742 & 3.9542 \\
\hline 9 & 9 & 97.0864 & 4.48578 & 92.5078 & 5.04474 & 4.5786 & 97.0864 & 0 & 95.3174 & 2.8096 \\
\hline 7 & 7 & 95.3174 & 3.85111 & 89.4901 & 4.2 & 5.8273 & 95.3174 & 0 & 93.3403 & 3.8502 \\
\hline Mean & & 97.37774 & & 93.17378 & & 4.20396 & & 0.02082 & & 3.14256 \\
\hline
\end{tabular}

Table A10. Scenario 4 - UL Optimised (ASBTBA).

\begin{tabular}{|c|c|c|c|c|c|c|c|c|c|c|}
\hline Num & Num & DL \% & DL & UL \% & UL & Covg & $\operatorname{Max} \%$ & Max DL & Max \% & Max UL \\
\hline $\mathrm{Tx}$ & Sites & Covg & Load & Covg & Load & Diff & DL Covg & Covg Diff & UL Covg & Covg Diff \\
\hline 15 & 11 & 98.0229 & 3.93876 & 98.231 & 7.83294 & 0.2081 & 98.3351 & 0.3122 & 98.231 & 0 \\
\hline 13 & 11 & 98.0229 & 4.08886 & 97.9188 & 7.36299 & 0.1041 & 98.4391 & 0.4162 & 97.9188 & 0 \\
\hline 11 & 10 & 97.1904 & 3.7737 & 96.7742 & 6.17889 & 0.4162 & 97.8148 & 0.6244 & 96.7742 & 0 \\
\hline 9 & 9 & 95.6296 & 3.40389 & 95.1093 & 5.27193 & 0.5203 & 97.0864 & 1.4568 & 95.3174 & 0.2081 \\
\hline 7 & 7 & 94.589 & 3.03997 & 93.3403 & 4.16951 & 1.2487 & 95.3174 & 0.7284 & 93.3403 & 0 \\
\hline Mean & & 96.69096 & & 96.27472 & & 0.49948 & & 0.7076 & & 0.04162 \\
\hline
\end{tabular}

Table A11. Scenario 5a - DL Optimised (ASBTBA).

\begin{tabular}{|c|c|c|c|c|c|c|c|c|c|c|}
\hline Num & Num & DL \% & DL & UL \% & UL & Covg & $\operatorname{Max} \%$ & Max DL & $\operatorname{Max} \%$ & Max UL \\
\hline $\mathrm{Tx}$ & Sites & Covg & Load & Covg & Load & Diff & DL Covg & Covg Diff & UL Covg & Covg Diff \\
\hline 30 & 12 & 95.7336 & 8.8114 & 92.2997 & 13.69 & 3.4339 & 95.7736 & 0.04 & 94.7971 & 2.4974 \\
\hline 28 & 12 & 95.0052 & 8.15436 & 90.5307 & 12.7677 & 4.4745 & 95.0052 & 0 & 94.3809 & 3.8502 \\
\hline 26 & 12 & 95.1093 & 8.94051 & 88.9698 & 12.7132 & 6.1395 & 95.1093 & 0 & 93.9646 & 4.9948 \\
\hline 24 & 12 & 94.7971 & 9.22445 & 87.513 & 10.9784 & 7.2841 & 94.7971 & 0 & 92.2591 & 4.7461 \\
\hline 22 & 12 & 93.9646 & 8.67947 & 84.2872 & 11.1338 & 9.6774 & 93.9646 & 0 & 91.155 & 6.8678 \\
\hline Mean & & 94.92196 & & 88.72008 & & 6.20188 & & 0.008 & & 4.59126 \\
\hline
\end{tabular}

Table A12. Scenario 5a - UL Optimised (ASBTBA).

\begin{tabular}{|c|c|c|c|c|c|c|c|c|c|c|}
\hline Num & Num & DL \% & DL & UL \% & UL & Covg & $\operatorname{Max} \%$ & Max DL & $\operatorname{Max} \%$ & Max UL \\
\hline$\overline{T x}$ & Sites & Covg & Load & Covg & Load & Diff & DL Covg & Covg Diff & UL Covg & Covg Diff \\
\hline 30 & 12 & 94.2768 & 14.8372 & 94.7971 & 16.3076 & 0.5203 & 95.7736 & 1.4968 & 94.7971 & 0 \\
\hline 28 & 11 & 93.9646 & 7.50289 & 94.3809 & 15.5006 & 0.4163 & 95.0052 & 1.0406 & 94.3809 & 0 \\
\hline 26 & 11 & 93.8606 & 7.97812 & 93.9646 & 14.5485 & 0.104 & 95.1093 & 1.2487 & 93.9646 & 0 \\
\hline 24 & 11 & 92.4037 & 12.1858 & 91.8835 & 13.4864 & 0.5202 & 94.7971 & 2.3934 & 92.2591 & 0.3756 \\
\hline 22 & 11 & 92.6119 & 8.14166 & 91.155 & 12.9164 & 1.4569 & 93.9646 & 1.3527 & 91.155 & 0 \\
\hline Mean & & 93.42352 & & 93.23622 & & 0.60354 & & 1.50644 & & 0.07512 \\
\hline
\end{tabular}


Table A13. Scenario 5b - DL Optimised (ASBTBA).

\begin{tabular}{|c|c|c|c|c|c|c|c|c|c|c|}
\hline Num & Num & DL \% & $\mathrm{DL}$ & UL \% & UL & Covg & $\operatorname{Max} \%$ & Max DL & Max \% & Max UL \\
\hline$\overline{T x}$ & Sites & Covg & Load & Covg & Load & Diff & DL Covg & Covg Diff & UL Covg & Covg Diff \\
\hline 30 & 12 & 94.3809 & 6.92736 & 93.1322 & 15.2262 & 1.2487 & 94.693 & 0.3121 & 94.7971 & 1.6649 \\
\hline 28 & 12 & 95.2133 & 8.13099 & 93.7565 & 14.1049 & 1.4568 & 95.2133 & 0 & 94.1727 & 0.4162 \\
\hline 26 & 12 & 94.7971 & 8.19764 & 91.051 & 15.6 & 3.7461 & 94.7971 & 0 & 94.849 & 3.798 \\
\hline 24 & 12 & 94.1727 & 7.94107 & 92.4037 & 13.511 & 1.769 & 94.1727 & 0 & 92.7159 & 0.3122 \\
\hline 22 & 12 & 93.7565 & 8.33987 & 88.5536 & 13.2 & 5.2029 & 93.7565 & 0 & 90.9469 & 2.3933 \\
\hline Mean & & 94.46410 & & 91.77940 & & 2.68470 & & 0.06242 & & 1.71692 \\
\hline
\end{tabular}

Table A14. Scenario 5b - UL Optimised (ASBTBA).

\begin{tabular}{|c|c|c|c|c|c|c|c|c|c|c|}
\hline Num & Num & DL \% & $\mathrm{DL}$ & UL \% & UL & Covg & Max \% & Max DL & Max \% & Max UL \\
\hline $\mathrm{Tx}$ & Sites & Covg & Load & Covg & Load & Diff & DL Covg & Covg Diff & UL Covg & Covg Diff \\
\hline 30 & 12 & 93.7565 & 14.4344 & 94.589 & 15.9992 & 0.8325 & 94.693 & 0.9365 & 94.7971 & 0.2081 \\
\hline 28 & 12 & 93.6524 & 7.0646 & 94.1727 & 16.0077 & 0.5203 & 95.2133 & 1.5609 & 94.1727 & 0 \\
\hline 26 & 12 & 93.8606 & 6.80798 & 94.849 & 14.5496 & 0.9884 & 94.7971 & 0.9365 & 94.849 & 0 \\
\hline 24 & 12 & 93.1322 & 7.31611 & 92.7159 & 13.1161 & 0.4163 & 94.1727 & 1.0405 & 92.7159 & 0 \\
\hline 22 & 11 & 91.3632 & 11.3738 & 90.9469 & 12.3443 & 0.4163 & 93.7565 & 2.3933 & 90.9469 & 0 \\
\hline Mean & & 93.15298 & & 93.45470 & & 0.63476 & & 1.37354 & & 0.04162 \\
\hline
\end{tabular}

Table A15. Scenario 5c - DL Optimised (ASBTBA).

\begin{tabular}{|c|c|c|c|c|c|c|c|c|c|c|}
\hline Num & Num & DL \% & DL & UL \% & UL & Covg & $\operatorname{Max} \%$ & Max DL & $\operatorname{Max} \%$ & Max UL \\
\hline $\mathrm{Tx}$ & Sites & Covg & Load & Covg & Load & Diff & DL Covg & Covg Diff & UL Covg & Covg Diff \\
\hline 30 & 12 & 95.2133 & 8.6136 & 91.155 & 18 & 4.0583 & 95.2133 & 0 & 94.7971 & 3.6421 \\
\hline 28 & 12 & 94.7971 & 8.33628 & 92.5078 & 13.8027 & 2.2893 & 94.7971 & 0 & 94.9011 & 2.3933 \\
\hline 26 & 12 & 94.4849 & 8.44079 & 91.155 & 12.5475 & 3.3299 & 94.4849 & 0 & 93.6524 & 2.4974 \\
\hline 24 & 12 & 94.693 & 9.255303 & 89.8023 & 11.7329 & 4.8907 & 94.693 & 0 & 92.4037 & 2.6014 \\
\hline 22 & 12 & 94.0687 & 9.59018 & 87.0968 & 10.4895 & 6.9719 & 94.0687 & 0 & 92.6119 & 5.5151 \\
\hline Mean & & 94.65140 & & 90.34338 & & 4.30802 & & 0 & & 3.32986 \\
\hline
\end{tabular}

Table A16. Scenario 5c - UL Optimised (ASBTBA).

\begin{tabular}{|c|c|c|c|c|c|c|c|c|c|c|}
\hline Num & Num & DL \% & $\mathrm{DL}$ & UL \% & $\mathrm{UL}$ & Covg & $\operatorname{Max} \%$ & Max DL & $\operatorname{Max} \%$ & Max UL \\
\hline$\overline{T x}$ & Sites & Covg & Load & Covg & Load & Diff & DL Covg & Covg Diff & UL Covg & Covg Diff \\
\hline 30 & 12 & 94.693 & 8.98932 & 94.7971 & 16.1597 & 0.1041 & 95.2133 & 0.5203 & 94.7971 & 0 \\
\hline 28 & 12 & 94.589 & 8.86264 & 94.9011 & 15.5587 & 0.3121 & 94.7971 & 0.2081 & 94.9011 & 0 \\
\hline 26 & 11 & 93.5484 & 8.20557 & 93.6524 & 14.5616 & 0.104 & 94.4849 & 0.9365 & 93.6524 & 0 \\
\hline 24 & 11 & 93.2362 & 8.83864 & 92.0916 & 12.937 & 1.1446 & 94.693 & 1.4568 & 92.4037 & 0.3121 \\
\hline 22 & 11 & 92.924 & 7.51445 & 92.6119 & 12.8082 & 0.3121 & 94.0687 & 1.1447 & 92.6119 & 0 \\
\hline Mean & & 93.79812 & & 93.61082 & & 0.39538 & & 0.85328 & & 0.06242 \\
\hline
\end{tabular}

Table A17. Scenario 6 - DL Optimised (ASBTBA).

\begin{tabular}{|c|c|c|c|c|c|c|c|c|c|c|}
\hline Num & Num & DL \% & DL & UL \% & UL & Covg & $\operatorname{Max} \%$ & Max DL & Max \% & Max UL \\
\hline $\mathrm{Tx}$ & Sites & Covg & Load & Covg & Load & Diff & DL Covg & Covg Diff & UL Covg & Covg Diff \\
\hline 30 & 12 & 76.5117 & 16.5578 & 53.5609 & 14.0817 & 22.9508 & 76.5117 & 0 & 60.602 & 7.0411 \\
\hline 28 & 12 & 75.6248 & 15.7692 & 52.3246 & 13.2735 & 23.3002 & 75.6248 & 0 & 58.8283 & 6.5037 \\
\hline 26 & 12 & 75.6517 & 14.9694 & 53.9371 & 13.9292 & 21.7146 & 75.6517 & 0 & 57.4308 & 3.4937 \\
\hline 24 & 12 & 78.8723 & 13.9065 & 51.5453 & 12.5168 & 27.327 & 78.8723 & 0 & 57.4577 & 5.9124 \\
\hline 22 & 12 & 73.4211 & 12.9731 & 50.1478 & 11.526 & 23.2733 & 73.4211 & 0 & 54.9583 & 4.8105 \\
\hline Mean & & 76.01632 & & 52.30314 & & 23.71318 & & 0 & & 5.55228 \\
\hline
\end{tabular}

Table A18. Scenario 6 - UL Optimised (ASBTBA).

\begin{tabular}{|c|c|c|c|c|c|c|c|c|c|c|}
\hline Num & Num & DL \% & DL & UL \% & $\mathrm{UL}$ & Covg & Max \% & Max DL & Max \% & Max UL \\
\hline $\mathrm{Tx}$ & Sites & Covg & Load & Covg & Load & Diff & DL Covg & Covg Diff & UL Covg & Covg Diff \\
\hline 30 & 12 & 74.308 & 15.7713 & 60.602 & 17.7095 & 13.706 & 76.5117 & 2.2037 & 60.602 & 0 \\
\hline 28 & 12 & 73.3405 & 14.5942 & 58.8283 & 16.0287 & 14.5122 & 75.6248 & 2.2843 & 58.8283 & 0 \\
\hline 26 & 12 & 71.6743 & 14.0077 & 57.4308 & 15.2955 & 14.2435 & 75.6517 & 3.9774 & 57.4308 & 0 \\
\hline 24 & 12 & 71.5937 & 13.1524 & 57.4577 & 14.4 & 14.136 & 78.8723 & 7.2786 & 57.4577 & 0 \\
\hline 22 & 12 & 69.9543 & 12.3729 & 54.9583 & 13.0276 & 14.996 & 73.4211 & 3.4668 & 54.9583 & 0 \\
\hline Mean & & 72.17416 & & 57.85542 & & 14.31874 & & 3.84216 & & 0 \\
\hline
\end{tabular}

